International Journal of Pure and Applied Mathematics

Volume 105 No. 3 2015, 359-376

ISSN: 1311-8080 (printed version); ISSN: 1314-3395 (on-line version)

url: http://www.ijpam.eu

doi: http://dx.doi.org/10.12732/ijpam.v105i3.4

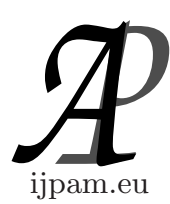

\title{
QUASI-BOUNDARY VALUE METHOD \\ FOR AN ILL POSED DIFFUSION SYSTEM
}

\author{
Sebti Djemoui ${ }^{1}$, Fairouz Zouyed ${ }^{2} \S$ \\ 1,2 Applied Mathematics Laboratory \\ University Badji Mokhtar Annaba \\ P.O. Box 12, El Hadjar, Annaba, 23000, ALGERIA
}

\begin{abstract}
In this paper we investigate an ill posed diffusion system with a non diagonal diffusion matrix. Based on the quasi-boundary value method, we regularize the problem, we prove that the approximate solutions depend continuously on the data and we establish some convergence results. Finally numerical results are presented to illustrate the accuracy and efficiency of the proposed method.
\end{abstract}

AMS Subject Classification: 35F 35, 65F22

Key Words: system of partial differential equations, ill-posed problem, regularization

\section{Introduction}

In this paper we consider the inverse problem of determining the source term $u(0, x)$ in a system of partial differential equations of the form

$$
\left\{\begin{array}{l}
u_{t}(t, x)-D \triangle u(t, x)=0, \quad 0<t<T, \quad x \in \Omega, \\
u(t, x)=0, \quad 0 \leq t<T, \quad x \in \partial \Omega, \\
u(T, x)=f(x), \quad x \in \Omega,
\end{array}\right.
$$

where $\Omega$ is a sufficiently regular bounded domain in $\mathbf{R}^{N}$ and $D$ is an $n \times n$

Received: July 5, 2015

(C) 2015 Academic Publications, Ltd.

$\S_{\text {Correspondence author }}$ 
real matrix with semi-simple and positive eigenvalues. The diffusion equation together with their modified forms and systems of diffusion equations have many important applications in mathematical physics, mathematical finance, chemistry, biology and environmental science [2], [3] ,[5], [6], [7]. In this study as previously mentioned, our purpose is to identify $u(0, x)$ from the final data $u(T, x)$, this problem is ill posed, even a unique solution exists it does not depend continuously on the data. Hence, a regularization is in order. In the mathematical literature various methods have been proposed for solving illposed problems, we can notably mention the quasi-boundary value method (QBVM). It has been used by many authors, such as L.S. Abdulkerimov [1], P.N. Vabishchevich et al [15], I.V. Melnikova et al [8], [11], [12], R.E. Showalter [14], G.W. Clark and S.F. Oppenheimer [4] and it has been successfully applied to various classes of elliptic and parabolic ill-posed problems.

In the present work, we extend the QBVM to systems of partial differential equations of the form (1.1) The study is based on the semigroups theory precisely the characterization of the $C_{0^{-}}$semigroup generated by the system (1.1) which is given and analyzed by Leiva [9] and Oliveira [10].

We note that though the diffusion systems forward in time have been extensively studied in literature, the case of diffusion systems backward in time does not seem have been widely investigated in spite of their physical and practical importance.

The paper is organized as follows, in Section 2 we introduce some preliminary results, Section 3 gives an abstract formulation of the problem and shows the ill posed-ness of the problem. In Section 4, we introduce the regularized solution and we give some convergence results. Finally numerical implementation is described in Section 5.

\section{Preliminaries}

Let $\mathcal{H}=L^{2}(\Omega)$ with the inner product $(.,$.$) and consider the following classical$ boundary-eigenvalue problem for the laplacien:

$$
\left\{\begin{array}{ccc}
-\Delta u=\lambda u, & \text { on } & \Omega, \\
u=0, & \text { on } & \partial \Omega,
\end{array}\right.
$$

where $\Omega$ is sufficiently regular bounded domain in $\mathbf{R}^{N}(N \geq 1)$, and $\mathcal{D}(-\Delta)=$ $\mathcal{H}^{2}(\Omega) \cap \mathcal{H}_{0}^{1}(\Omega)$. The problem $(2.1)$ has a countable set of eigenvalues

$$
0<\lambda_{1}<\lambda_{2}<\lambda_{3}<\ldots<\lambda_{j} \rightarrow+\infty \text { as } j \rightarrow+\infty .
$$


Each eigenvalue $\lambda_{j}$ with finite multiplicity $\gamma_{j}$ equal to the dimension of the corresponding eigenspace $S_{j}$.

1. Therefore, there exists a complete orthonormal set $\left\{\varphi_{j k}\right\}_{k=1}^{k=\gamma_{j}}$ of eigenvectors of $-\Delta$ such that, $\bullet$ for all $w \in \mathcal{D}(-\Delta)$, we have

$$
-\Delta w=\sum_{j=1}^{\infty} \lambda_{j} E_{j} w
$$

where

$$
E_{j} w=\sum_{k=1}^{\gamma_{j}}\left(w, \varphi_{j k}\right) \varphi_{j k} .
$$

So, $\left\{E_{j}\right\}$ is a family of complete orthogonal projections in $\mathcal{H}$ and for all $w \in \mathcal{H}$, we have $w=\sum_{j=1}^{\infty} E_{j} w$.

2. The operator $\Delta$ generates an analytic semigroup $\{\mathcal{T}(t)\}_{t \geq 0}$ on $\mathcal{H}$, defined by

$$
\mathcal{T}(t) w=\sum_{j=1}^{\infty} e^{-\lambda_{j} t} E_{j} w .
$$

Now, we denote by $Z$ the Hilbert space $\left(L^{2}(\Omega)\right)^{n}$ of the square integrable functions $u: \Omega \rightarrow \mathbf{R}^{N}$ with the usual inner product

$$
\langle u, v\rangle=\int_{\Omega}\left(u_{1}(x) \overline{v_{1}}(x)+\ldots .+u_{n}(x) \overline{v_{n}}(x)\right) d x .
$$

We define the following unbounded operator

$$
\begin{gathered}
A: \mathcal{D}(A) \subset Z \rightarrow Z, \\
A u=-D \Delta u, u \in \mathcal{D}(A),
\end{gathered}
$$

with

$$
\mathcal{D}(A)=\left(\mathcal{H}^{2}(\Omega) \cap \mathcal{H}_{0}^{1}(\Omega)\right)^{n} .
$$

Therefore, for all $u \in \mathcal{D}(A)$ we obtain,

$$
A u=\sum_{j=1}^{\infty} \lambda_{j} D P_{j} u
$$

and

$$
u=\sum_{j=1}^{\infty} P_{j} u,\|u\|^{2}=\sum_{j=1}^{\infty}\left\|P_{j} u\right\|^{2}, \quad u \in Z
$$


where

$$
P_{j}=\operatorname{diag}\left(E_{j}, E_{j}, \ldots, E_{j}\right)
$$

is a family of complete orthogonal projections in $Z$.

Theorem 2.1. [10, Theorem 1, p. 2] Assume that $d>0$ for all $d \in \sigma(D)$. Then

1. $A$ is a sectorial operator and therefore, $-A$ is the infinitesimal generator of an analytic $C_{0}$-semigroup $\{S(t)\}_{t \geq 0}$, on $Z$.

$$
S(t) x=\sum_{j=1}^{\infty} e^{A_{j} t} P_{j} x, \quad t \geq 0, \quad x \in Z,
$$

where $A_{j}=-\lambda_{j} D$.

2. $\{S(t)\}_{t \geq 0}$ is a compact $C_{0}$-semigroup in $\mathcal{L}(Z)$ and there exist constants $C \geq 1$ and $\beta>0$ such that

$$
\left\|e^{-A t}\right\|_{\mathcal{L}(Z)} \leq C e^{-\beta t}, \text { for all } t \geq 0 .
$$

\section{Ill-Posedness of the Problem}

In the Hilbert space $Z$, the system (1.1) can be written as an abstract functional differential equation

$$
\left\{\begin{array}{c}
u_{t}(t)+A u(t)=0, \quad 0<t<T, \\
u(T)=f, \quad f \in Z .
\end{array}\right.
$$

Let us consider the following direct problem corresponding to the backward Cauchy problem (3.1)

$$
\left\{\begin{array}{c}
v_{t}(t)+A v(t)=0, \quad 0<t<T \\
v(0)=g, \quad g \in Z
\end{array}\right.
$$

Since $A$ is the generator of an analytic semigroup, then for all $g \in Z$, the problem (3.2) has a unique solution $v \in C([0, T], Z)$ given by

$$
v(t)=S(t) g=\sum_{j=1}^{\infty} e^{-\lambda_{j} D t} P_{j} g,
$$

where $g=\sum_{j=1}^{\infty} P_{j} g$, see [13], Chap. 4, theorem 1.4, p. 104). 
Remark The matrix $D$ does not necessarily have distinct eigenvalues. Let $0<d_{1}<\ldots<d_{s}, s \leq n$ be the distinct eigenvalues, then $D$ admits the following spectral decomposition

$$
D=\sum_{i=1}^{s} d_{i} \mathcal{Q}_{i}
$$

where $\left\{\mathcal{Q}_{i}\right\}_{1 \leq i \leq s}$ is a complete family of projections.

Then the solution of the problem (3.2) can be written as follows

$$
v(t)=\sum_{j=1}^{\infty} e^{-\lambda_{j} D t} P_{j} g=\sum_{j=1}^{\infty}\left(\sum_{i=1}^{s} e^{-\lambda_{j} d_{i} t} \mathcal{Q}_{i}\right) P_{j} g .
$$

Theorem 3.1. The problem (3.1) admits a solution if and only

$$
\sum_{j=1}^{\infty}\left\|e^{\lambda_{j} D T} P_{j} f\right\|^{2}=\sum_{j=1}^{\infty}\left\|\sum_{i=1}^{s} e^{\lambda_{j} d_{i} T} \mathcal{Q}_{i} P_{j} f\right\|^{2}<\infty .
$$

Proof. If the problem (3.1) admits a solution $u$ then $u(t)=S(t) u(0)$.

So, $f=u(T)=S(T) u(0)=e^{-T A} u(0)$. It follows that

$$
\|u(0)\|^{2}=\sum_{j=1}^{\infty}\left\|e^{\lambda_{j} D T} P_{j} f\right\|^{2}<\infty .
$$

Now, if we get (3.4) we can define

$$
w=\sum_{j=1}^{\infty} \sum_{i=1}^{s} e^{\lambda_{j} d_{i} T} \mathcal{Q}_{i} P_{j} f \in Z,
$$

and consider the problem

$$
\left\{\begin{array}{l}
u_{t}(t)+A u(t)=0, \quad 0<t<T, \\
u(0)=w .
\end{array}\right.
$$

Since (3.5) is the direct well-posed, so it has a unique solution given by

$$
u(t)=S(t) w=\sum_{j=1}^{\infty} \sum_{i=1}^{s} e^{\lambda_{j} d_{i}(T-t)} \mathcal{Q}_{i} P_{j} f=\sum_{j=1}^{\infty} e^{\lambda_{j} D(T-t)} P_{j} f .
$$


Let $t=T$ in (3.6), we obtain

$$
u(T)=\sum_{j=1}^{\infty} P_{j} f=f .
$$

Hence, $u$ is the unique solution to (3.1).

Since $t<T$, we can see from (3.6) that the terms $e^{\lambda_{j} d_{i}(T-t)}$ are the instability causes. In the following section, it is our aim to restore the stability of the problem (3.1) by using a regularization method.

\section{Quasi-Boundary Value Method}

We shall regularize problem (3.1) using the quasi-boundary value method. Let us consider the approximate problem

$$
\left\{\begin{array}{cc}
u_{\alpha}^{\prime}(t)+A u_{\alpha}(t)=0, & 0<t<T, \\
\alpha u_{\alpha}(0)+u_{\alpha}(T)=f, & f \in Z
\end{array}\right.
$$

Definition 4.1. Define

$$
\begin{aligned}
u_{\alpha}(t) & =S(t)(\alpha I+S(T))^{-1} f \\
& =\sum_{j=1}^{\infty} e^{-\lambda_{j} D t}\left(\alpha I_{n}+e^{-\lambda_{j} D T}\right)^{-1} P_{j} f \\
& =\sum_{j=1}^{\infty} \sum_{i=1}^{s} e^{-\lambda_{j} d_{i} t}\left(\alpha+e^{-\lambda_{j} d_{i} T}\right)^{-1} \mathcal{Q}_{i} P_{j} f,
\end{aligned}
$$

for $f \in Z, \alpha>0$ and $t \in[0, T]$.

Theorem 4.1. The function $u_{\alpha}(t)$ is the unique solution of (4.1) and it depends continuously on $f$.

Proof. Consider the problem:

$$
\left\{\begin{array}{l}
w_{\alpha}^{\prime}(t)+A w_{\alpha}(t)=0, \quad 0<t<T \\
w_{\alpha}(0)=f_{\alpha}
\end{array}\right.
$$

where $f_{\alpha}=(\alpha I+S(T))^{-1} f$. 
This problem is well-posed and its solution is given by

$$
w_{\alpha}(t)=S(t) f_{\alpha}=S(t)(\alpha I+S(T))^{-1} f .
$$

Observe that

$$
\begin{aligned}
w_{\alpha}(T)+\alpha w_{\alpha}(0) & =S(T)(S(T)+\alpha I)^{-1} f+\alpha(S(T)+\alpha I)^{-1} f \\
& =(S(T)+\alpha I)(S(T)+\alpha I)^{-1} f=f .
\end{aligned}
$$

Thanks to (4.5) and uniqueness of solution to direct problem (4.3), we deduce that the problem (4.1) admits the unique solution $u_{\alpha}$ given by (4.2).

To show the continuous dependence of $u_{\alpha}$ on $f$, we compute

$$
\begin{aligned}
\left\|u_{\alpha}(t)\right\|^{2} & =\left\|\sum_{j=1}^{\infty} e^{-\lambda_{j} D t}\left(\alpha I_{n}+e^{-\lambda_{j} D T}\right)^{-1} P_{j} f\right\|^{2} \\
& =\sum_{j=1}^{\infty}\left\|\sum_{i=1}^{s} e^{-\lambda_{j} d_{i} t}\left(\alpha+e^{-\lambda_{j} d_{i} T}\right)^{-1} \mathcal{Q}_{i} P_{j} f\right\|^{2} \\
& \leq \sum_{j=1}^{\infty}\left\|\left(\sum_{i=1}^{s} e^{-\lambda_{j} d_{i} t}\left(\alpha+e^{-\lambda_{j} d_{i} T}\right)^{-1} \mathcal{Q}_{i}\right)\right\|^{2}\left\|P_{j} f\right\|^{2} \\
& \leq \sum_{j=1}^{\infty}\left(\sum_{i=1}^{s} e^{-\lambda_{j} d_{i} t}\left(\alpha+e^{-\lambda_{j} d_{i} T}\right)^{-1}\left\|\mid \mathcal{Q}_{i}\right\|\right)^{2}\left\|P_{j} f\right\|^{2} \\
& \leq \sum_{j=1}^{\infty}\left(\sum_{i=1}^{s}\left(\alpha+e^{-\lambda_{j} d_{i} T}\right)^{-1}\left\|\left|\mathcal{Q}_{i}\right|\right\|\right)^{2}\left\|P_{j} f\right\|^{2} \\
& \leq \frac{1}{\alpha^{2}} \sum_{j=1}^{\infty}\left(\sum_{i=1}^{s}\left\|\left|\mathcal{Q}_{i}\right|\right\|\right)^{2}\left\|P_{j} f\right\|^{2} \\
& \leq \frac{1}{\alpha^{2}} M^{2} \sum_{j=1}^{\infty}\left\|P_{j} f\right\|^{2} \\
& \leq \frac{1}{\alpha^{2}} M^{2}\|f\|^{2}
\end{aligned}
$$

where $M=\sum_{i=1}^{s}\left\|\left|\mathcal{Q}_{i}\right|\right\|,(\||\cdot|\|$ is norm matrix.)

Theorem 4.2. For all $f \in Z, \alpha>0$ and $t \in[0, T]$, we have that

$$
\left\|u_{\alpha}(t)\right\| \leq \alpha^{\frac{t}{T}-1} M\|f\| .
$$


Proof. Let $f=\sum_{j=1}^{\infty} P_{j} f$, then

$$
\begin{aligned}
\left\|u_{\alpha}(t)\right\|^{2} & =\left\|\sum_{j=1}^{\infty} e^{-\lambda_{j} D t}\left(\alpha I+e^{-\lambda_{j} D T}\right)^{-1} P_{j} f\right\|^{2} \\
& =\sum_{j=1}^{\infty}\left\|\sum_{i=1}^{s} e^{-\lambda_{j} d_{i} t}\left(\alpha+e^{-\lambda_{j} d_{i} T}\right)^{-1} \mathcal{Q}_{i} P_{j} f\right\|^{2} \\
& \leq \sum_{j=1}^{\infty}\left(\sum_{i=1}^{s} e^{-\lambda_{j} d_{i} t}\left\{\left(\alpha+e^{-\lambda_{j} d_{i} T}\right)^{\frac{t}{T}}\left(\alpha+e^{-\lambda_{j} d_{i} T}\right)^{1-\frac{t}{T}}\right\}^{-1}\left\|\mathcal{Q}_{i} \mid\right\|\right)^{2}\left\|P_{j} f\right\|^{2} \\
& \leq \sum_{j=1}^{\infty}\left(\sum_{i=1}^{s}\left(\alpha+e^{-\lambda_{j} d_{i} T}\right)^{-\left(1-\frac{t}{T}\right)}\left\|\left|\mathcal{Q}_{i}\right|\right\|\right)^{2}\left\|P_{j} f\right\|^{2} \\
& \leq\left(\frac{1}{\alpha^{2}}\right)^{1-\frac{t}{T}} \sum_{j=1}^{\infty}\left(\sum_{i=1}^{s}\left\|\left|\mathcal{Q}_{i}\right|\right\|\right)^{2}\left\|P_{j} f\right\|^{2} \\
& \leq\left(\frac{1}{\alpha^{2}}\right)^{1-\frac{t}{T}} M^{2} \sum_{j=1}^{\infty}\left\|P_{j} f\right\|^{2},
\end{aligned}
$$

which implies that

$$
\left\|u_{\alpha}(t)\right\| \leq \alpha^{\frac{t}{T}-1} M\|f\|
$$

Theorem 4.3. For all $f \in Z, u_{\alpha}(T)$ converges to $f$ in $Z$ as $\alpha$ tends to zero.

Proof. Let $\sum_{j=1}^{\infty} P_{j} f$, then

$$
\begin{aligned}
\left\|u_{\alpha}(T)-f\right\|^{2} & =\sum_{j=1}^{\infty}\left\|\left[e^{-\lambda_{j} D T}\left(\alpha I_{n}+e^{-\lambda_{j} D T}\right)^{-1}-I_{n}\right] P_{j} f\right\|^{2} \\
& =\sum_{j=1}^{\infty}\left\|\sum_{i=1}^{s}\left(e^{-\lambda_{j} d_{i} T}\left(\alpha+e^{-\lambda_{j} d_{i} T}\right)^{-1}-1\right) \mathcal{Q}_{i} P_{j} f\right\|^{2} \\
& =\sum_{j=1}^{\infty}\left\|\sum_{i=1}^{s} \alpha\left(\alpha+e^{-\lambda_{j} d_{i} T}\right)^{-1} \mathcal{Q}_{i} P_{j} f\right\|^{2} \\
& \leq \sum_{j=1}^{\infty}\left(\sum_{i=1}^{s} \alpha\left(\alpha+e^{-\lambda_{j} d_{i} T}\right)^{-1}\left\|\left|\mathcal{Q}_{i}\right|\right\|\right)^{2}\left\|P_{j} f\right\|^{2}
\end{aligned}
$$


Fix $\varepsilon>0$ and choose $\aleph$ so that $M^{2} \sum_{j=\aleph+1}^{\infty}\left\|P_{j} f\right\|^{2}<\varepsilon / 2$. Thus

$$
\begin{aligned}
\left\|u_{\alpha}(T)-f\right\|^{2} & \leq \sum_{j=1}^{\aleph}\left(\sum_{i=1}^{s} \alpha\left(\alpha+e^{-\lambda_{j} d_{i} T}\right)^{-1}\left\|\left|\mathcal{Q}_{i}\right|\right\|\right)^{2}\left\|P_{j} f\right\|^{2} \\
& +\sum_{j=\aleph+1}^{\infty}\left(\sum_{i=1}^{s} \alpha\left(\alpha+e^{-\lambda_{j} d_{i} T}\right)^{-1}\left\|\left|\mathcal{Q}_{i}\right|\right\|\right)^{2}\left\|P_{j} f\right\|^{2} \\
& \leq \sum_{j=1}^{\aleph} \alpha^{2} e^{2 \lambda_{j} d_{s} T}\left(\sum_{i=1}^{s}\left\|\left|\mathcal{Q}_{i}\right|\right\|\right)^{2}\left\|P_{j} f\right\|^{2}+\sum_{j=\aleph+1}^{\infty}\left(\sum_{i=1}^{s}\left\|\mathcal{Q}_{i} \mid\right\|\right)^{2}\left\|P_{j} f\right\|^{2} \\
& \leq \alpha^{2} M^{2} \sum_{j=1}^{\aleph} e^{2 \lambda_{j} d_{s} T}\left\|P_{j} f\right\|^{2}+M^{2} \sum_{j=\aleph+1}^{\infty}\left\|P_{j} f\right\|^{2} . \\
& \leq \alpha^{2} M^{2} \sum_{j=1}^{\aleph} e^{2 \lambda_{j} d_{s} T}\left\|P_{j} f\right\|^{2}+\frac{\varepsilon}{2} .
\end{aligned}
$$

Now, let $\alpha$ be such that $\alpha^{2}<\varepsilon\left(2 M^{2} \sum_{j=1}^{\aleph} e^{2 \lambda_{j} d_{s} T}\left\|P_{j} f\right\|^{2}\right)^{-1}$.

Hence we are done.

Definition 4.2. Define the set

$$
C_{\theta}(A)=\left\{\phi \in Z,\|\phi\|_{\theta}^{2}=\sum_{j=1}^{+\infty} e^{2 T \theta \lambda_{j} d_{s}}\left\|P_{j} f\right\|^{2}<\infty\right\}, \quad \theta \geq 0 .
$$

Theorem 4.4. If there exists $0<\theta<2$, such that $f \in C_{\theta}(A)$ then $\left\|u_{\alpha}(T)-f\right\|$ converge to zero with order $\alpha^{\theta}$.

Proof. Let $k \in(0,2)$. Fix natural number $j$, put $\gamma_{j}=\lambda_{j} d_{s}$, and

$$
h_{j}(\alpha)=\frac{\alpha^{k}}{\left(\alpha+e^{-\gamma_{j} T}\right)^{2}},
$$

Differentiating with respect to $\alpha$, once obtain

$$
h_{j}^{\prime}(\alpha)=\alpha^{k-1} \frac{(k-2) \alpha+k e^{-\gamma_{j} T}}{\left(\alpha+e^{-\gamma_{j} T}\right)^{3}} .
$$

Thus $h_{j}^{\prime}(\alpha)=0$, if $\alpha=0$ or

$$
\alpha=\frac{k}{2-k} e^{-\gamma_{j} T} .
$$


Since $h_{j}(\alpha)>0, h_{j}(0)=0$ and $\lim _{\alpha \rightarrow 0} h_{j}(\alpha)=0$, we have that $\alpha^{\prime}=\frac{k}{2-k} e^{-\gamma_{j} T}$ is the critical point at which $h_{j}$ achieves its maximum. Thus we have

$$
\begin{aligned}
h_{j}(\alpha) \leq h_{j}\left(\alpha^{\prime}\right) & =\frac{\left(\frac{k}{2-k}\right)^{k} e^{-k T \gamma_{j}}}{\left(\alpha^{\prime}+e^{-\gamma_{j} T}\right)^{2}} \\
& \leq\left(\frac{k}{2-k}\right)^{k} e^{(2-k) T \gamma_{j}}\left(\alpha^{\prime 2}+2 \alpha^{\prime} e^{\gamma_{j} T}+1\right)^{-1} \\
& \leq\left(\frac{k}{2-k}\right)^{k} e^{(2-k) T \gamma_{j}} .
\end{aligned}
$$

We calculate

$$
\begin{aligned}
\left\|u_{\alpha}(T)-f\right\|^{2} & \leq \sum_{j=1}^{\infty}\left(\sum_{i=1}^{s} \frac{\alpha}{\left(\alpha+e^{-\lambda_{j} d_{i} T}\right)}\left\|\mathcal{Q}_{i} \mid\right\|\right)^{2}\left\|P_{j} f\right\|^{2} \\
& \leq \sum_{j=1}^{\infty} \frac{\alpha^{2}}{\left(\alpha+e^{-\lambda_{j} d_{s} T}\right)^{2}}\left(\sum_{i=1}^{s}\left\|\left|\mathcal{Q}_{i}\right|\right\|\right)^{2}\left\|P_{j} f\right\|^{2} \\
& \leq \alpha^{2-k} M^{2} \sum_{j=1}^{\infty} h_{j}(\alpha)\left\|P_{j} f\right\|^{2} \\
& \leq \alpha^{2-k} M^{2} \sum_{j=1}^{\infty}\left(\frac{k}{2-k}\right)^{k} e^{(2-k) T \lambda_{j} d_{s}}\left\|P_{j} f\right\|^{2} .
\end{aligned}
$$

If we choose $k=2-\theta$, we obtain

$$
\left\|u_{\alpha}(T)-f\right\|^{2} \leq\left(\frac{2-\theta}{\theta}\right)^{2-\theta} \alpha^{\theta} M^{2} \sum_{j=1}^{\infty} e^{\theta T \lambda_{j} d_{s}}\left\|P_{j} f\right\|^{2} .
$$

This completes the proof.

Lemma 4.1. For all $f \in Z$, the problem (3.1) has a solution $u(t)$ if $u_{\alpha}(0)$ converges in $Z$. Furthermore, we then have that $u_{\alpha}(t)$ converges to $u(t)$ as the parameter $\alpha$ tends to zero uniformly in $t$.

Proof. Assume that $\lim _{\alpha \rightarrow 0} u_{\alpha}(0)=\xi$ exists and let $u(t)=S(t) \xi$. We compute

$$
\begin{aligned}
\left\|u_{\alpha}(t)-u(t)\right\| & =\left\|u_{\alpha}(t)-S(t) \xi\right\| \\
& \leq\|S(t)\|_{\mathcal{L}(Z)}\left\|(\alpha I+S(T))^{-1} f-\xi\right\| . \\
& \leq\|S(t)\|_{\mathcal{L}(Z)}\left\|u_{\alpha}(0)-\xi\right\| .
\end{aligned}
$$


Which implies that

$$
\sup _{0 \leq t \leq T}\left\|u_{\alpha}(t)-u(t)\right\| \leq C\left\|u_{\alpha}(0)-\xi\right\| \rightarrow 0, \text { as } \alpha \rightarrow 0 .
$$

Since $\lim _{\alpha \rightarrow 0} u_{\alpha}(T)=f$ and $\lim _{\alpha \rightarrow 0} u_{\alpha}(T)=u(T)$, then $u(T)=f$. Thus $u(t)=S(t) \xi$ solves the problem (3.1).

Theorem 4.5. Assume that the problem (3.1) admits a solution $u(t)$ and let $f \in C_{1}(A)$. Then $u_{\alpha}(0)$ converges in $Z$.

Proof. Since $f \in C_{1}(A)$, we can fix $\varepsilon>0$ and choose $\aleph$ so that

$$
M^{2} \sum_{j=1+\aleph}^{\infty} e^{2 \lambda_{j} d_{s} T}\left\|P_{j} f\right\|^{2}<\frac{\varepsilon}{2} .
$$

We compute

$$
\begin{aligned}
\left\|u_{\alpha}(0)-u(0)\right\|^{2} & =\sum_{j=1}^{\infty}\left\|\sum_{i=1}^{s}\left[\left(\alpha+e^{-\lambda_{j} d_{i} T}\right)^{-1}-e^{\lambda_{j} d_{i} T}\right] \mathcal{Q}_{i} P_{j} f\right\|^{2} \\
& =\sum_{j=1}^{\infty}\left\|\sum_{i=1}^{s}\left[\frac{\alpha e^{\lambda_{j} d_{i} T}}{\alpha+e^{-\lambda_{j} d_{i} T}}\right] \mathcal{Q}_{i} P_{j} f\right\|^{2} \\
& =I_{1}+I_{2}
\end{aligned}
$$

where

$$
\begin{aligned}
I_{1} & =\sum_{j=1}^{\aleph}\left\|\sum_{i=1}^{s}\left[\frac{\alpha e^{\lambda_{j} d_{i} T}}{\alpha+e^{-\lambda_{j} d_{i} T}}\right] \mathcal{Q}_{i} P_{j} f\right\|^{2} \\
& \leq \sum_{j=1}^{\aleph}\left(\sum_{i=1}^{s} e^{\lambda_{j} d_{i} T}\left(\frac{\alpha e^{\lambda_{j} d_{i} T}}{1+\alpha e^{\lambda_{j} d_{i} T}}\right)\left\|\mathcal{Q}_{i}\right\|\right)^{2}\left\|P_{j} f\right\|^{2} \\
& \leq \alpha^{2} \sum_{j=1}^{\aleph}\left(\sum_{i=1}^{s} e^{2 \lambda_{j} d_{i} T}\|\| \mathcal{Q}_{i} \|\right)^{2}\left\|P_{j} f\right\|^{2} \\
& \leq \alpha^{2} M^{2} \sum_{j=1}^{\aleph} e^{4 \lambda_{j} d_{s} T}\left\|P_{j} f\right\|^{2}
\end{aligned}
$$

and

$$
I_{2}=\sum_{j=\aleph+1}^{\infty}\left\|\sum_{i=1}^{s}\left[\frac{\alpha e^{\lambda_{j} d_{i} T}}{\alpha+e^{-\lambda_{j} d_{i} T}}\right] \mathcal{Q}_{i} P_{j} f\right\|^{2}
$$




$$
\begin{aligned}
& \leq \sum_{j=\aleph+1}^{\infty}\left(\sum_{i=1}^{s} e^{\lambda_{j} d_{i} T}\left(\frac{\alpha e^{\lambda_{j} d_{i} T}}{1+\alpha e^{\lambda_{j} d_{i} T}}\right)\left\|\left|\mathcal{Q}_{i}\right|\right\|\right)^{2}\left\|P_{j} f\right\|^{2} \\
& \leq \sum_{j=1+\aleph}^{\infty}\left(\sum_{i=1}^{s} e^{\lambda_{j} d_{i} T}\left\|\left|Q_{i}\right|\right\|\right)^{2} \mid\left\|P_{j} f\right\|^{2} \\
& \leq M^{2} \sum_{j=1+\aleph}^{\infty} e^{2 \lambda_{j} d_{s} T}\left\|P_{j} f\right\|^{2}<\frac{\varepsilon}{2} .
\end{aligned}
$$

If we choose $\alpha$ such that $\alpha^{2}<\varepsilon\left(2 M^{2} \sum_{j=1}^{\aleph} e^{4 \lambda_{j} d_{s} T}\left\|P_{j} f\right\|^{2}\right)^{-1}$, we obtain

$$
\left\|u_{\alpha}(0)-u(0)\right\|^{2}=I_{1}+I_{2}<\varepsilon .
$$

This shows that $u_{\alpha}(0)$ converges to $u(0)$ as $\alpha$ tends to zero.

Theorem 4.6. Assume that the problem (3.1) admits a solution $u(t)$. If there exists $0<\theta<2$ so that $f \in C_{\theta}(A)$, then $\left\|u_{\alpha}(0)-u(0)\right\|$ converges to zero with order $\alpha^{\theta}$.

Proof. Working as in the proof of theorem 4.5 we obtain

$$
\begin{aligned}
\left\|u_{\alpha}(0)-u(0)\right\|^{2} & \leq \alpha^{2-k} M^{2} \sum_{j=1}^{\infty} h_{j}(\alpha) e^{2 \lambda_{j} d_{s} T}\left(\sum_{i=1}^{s}\left\|\mid \mathcal{Q}_{i}\right\|\right)^{2}\left\|P_{j} f\right\|^{2} \\
& \leq \alpha^{2-k}\left(\frac{k}{2-k}\right)^{k} M^{2} \sum_{j=1}^{\infty} e^{(4-k) \lambda_{j} d_{s} T}\left\|P_{j} f\right\|^{2},
\end{aligned}
$$

as above, letting $k=2-\theta$ we obtain the result.

From the inequality (4.6) and theorem 4.6, we arrive at the following

Corollary 4.1. Assume that the problem (3.1) admits a solution $u(t)$. If there exists $0<\theta<2$ so that $f \in C_{\theta}(A)$, then $u_{\alpha}(t)$ converges to $u(t)$ as the parameter $\alpha$ tends to zero with order $\alpha^{\theta}$ uniformly in $t$.

Let us now construct a family of regularizing operators for the problem (3.1).

Definition 4.3. A family of bounded linear operators $R_{\alpha}(t): Z \rightarrow Z$, $\alpha>0, \quad t \in[0, T]$ is called a family of regularizing operators for the problem 
(3.1) if for each $u(t)(0 \leq t \leq T)$ solution of the problem (3.1)with the final element $f$, and for any $\delta>0$, there exists $\alpha(\delta)>0$, such that

1. $\alpha(\delta) \rightarrow 0, \quad \delta \rightarrow 0$,

2. $\left\|R_{\alpha(\delta)}(t) f_{\delta}-u(t)\right\| \rightarrow 0, \quad \delta \rightarrow 0$, for each $t \in[0, T]$, on condition that $f_{\delta}$ satisfies $\left\|f_{\delta}-f\right\| \leq \delta$.

Define $R_{\alpha}(t)=e^{-t A}\left(\alpha+e^{-T A}\right)^{-1}, \quad t \geq 0, \quad \alpha>0$. It is clear that $\left\{R_{\alpha}(t), t \in[0, T], \alpha>0\right\} \subset \mathcal{L}(Z)$.

Theorem 4.7. Assuming that $f$ satisfies (3.4). Then the family $\left\{R_{\alpha}(t)\right\}$ defined above is a family of regularizing operators for (3.1).

Proof. We have

$$
\left\|R_{\alpha}(t) f_{\delta}-u(t)\right\| \leq\left\|R_{\alpha}(t)\left(f_{\delta}-f\right)\right\|+\left\|R_{\alpha}(t) f-u(t)\right\|,
$$

where

$$
\left\|R_{\alpha}(t)\left(f_{\delta}-f\right)\right\| \leq \frac{1}{\alpha} \delta
$$

Choose $\alpha=\sqrt{\delta}$, then $\alpha(\delta) \rightarrow 0, \delta \rightarrow 0$, and

$$
\left\|R_{\alpha}(t)\left(f_{\delta}-f\right)\right\| \leq \sqrt{\delta} \rightarrow 0, \text { as } \delta \rightarrow 0 .
$$

On the other hand and by virtue of theorem 4.5 , we have

$$
\left\|R_{\alpha}(t) f-u(t)\right\|=\left\|u_{\alpha}(t)-u(t)\right\| \rightarrow 0 \text { as } \delta \rightarrow 0,
$$

uniformly in $t$. Combining (4.8) and (4.9) we obtain

$$
\sup _{0 \leq t \leq T}\left\|R_{\alpha}(t)\left(f_{\delta}-f\right)\right\| \leq \sqrt{\delta} \text {, as } \delta \rightarrow 0 .
$$

Then, we deduce that $\left\{R_{\alpha}(t)\right\}$ is a family of regularizing operators for (3.1).

Remark All the previous results obtained in this note remain true if

1. the matrix $D$ has semi-simple eigenvalues in the half plane $\operatorname{Re} \sigma(D)>0$.

2. the $-\triangle$ in (1.1) is replaced by a positive self-adjoint linear operator with compact resolvent. This consideration should be useful to study abstract versions of the problem. 


\section{Numerical Results}

In this section, an example is devised for verifying the effectiveness of the proposed method. Consider the operator

$$
\mathcal{B}=-\frac{\partial^{2}}{\partial x^{2}} \text {, with } \mathcal{D}(\mathcal{B})=H_{0}^{1}(0, \pi) \subset \mathcal{H}=L^{2}(0, \pi) .
$$

$\lambda_{j}=j^{2}, \phi_{j}=\sqrt{\frac{2}{\pi}} \sin (j x), j=1,2, \ldots$ are eigenvalues and orthonormal eigenfunctions, which form a basis for $\mathcal{H}$.

Let $D=\left(\begin{array}{cc}-1 & -3 \\ 2 & 4\end{array}\right)$, whose eigenvalues are $d_{1}=1$ and $d_{2}=2$.

$\left\{\mathcal{Q}_{1}, \mathcal{Q}_{2}\right\} \in\left(M_{2}(\mathbf{R})\right)^{2}$ is the set of complementary projections on $\mathbf{R}^{2}$,

$$
\mathcal{Q}_{1}=\left(\begin{array}{cc}
3 & 3 \\
-2 & -2
\end{array}\right), \quad \mathcal{Q}_{2}=\left(\begin{array}{cc}
-2 & -3 \\
2 & 3
\end{array}\right) \text {. }
$$

such that

$$
D=d_{1} \mathcal{Q}_{1}+d_{2} \mathcal{Q}_{2},
$$

hence

$$
e^{-\lambda_{j} D t}=e^{-\lambda_{j} d_{1} t} \mathcal{Q}_{1}+e^{-\lambda_{j} d_{2} t} \mathcal{Q}_{2} .
$$

Consider the problem of finding $U=\left(\begin{array}{l}u_{1} \\ u_{2}\end{array}\right)$ from the system

$$
\left\{\begin{array}{l}
\partial_{t} U(t, x)-\frac{\partial^{2}}{\partial x^{2}} U(t, x)=0, \quad(t, x) \in(0,1) \times(0, \pi) \\
U(t, 0)=U(t, \pi)=0, \quad, t \in(0,1) \\
U(1, x)=f(x), \quad x \in(0, \pi)
\end{array}\right.
$$

where $f(x)=\left(\begin{array}{c}\left(6 e^{-1}-5 e^{-2}\right) \sin x \\ \left(-4 e^{-1}+5 e^{-2}\right) \sin x\end{array}\right)$. The exact solution of this problem is

$$
U(t, x)=\left(\begin{array}{c}
\left(6 e^{-t}-5 e^{-2 t}\right) \sin x \\
\left(-4 e^{-t}+5 e^{-2 t}\right) \sin x
\end{array}\right) .
$$

To see the ill-posed nature of problem $(\mathcal{P})$, we consider the perturbed data function

$$
f_{m}(x)=\left(\begin{array}{c}
\left(6 e^{-1}-5 e^{-2}\right) \sin x+\frac{1}{m} \sin m x \\
\left(-4 e^{-1}+5 e^{-2}\right) \sin x+\frac{1}{m} \sin m x
\end{array}\right) .
$$

The exact solution of problem $(\mathcal{P})$ corresponding to the perturbed data is

$$
U_{m}(t, x)=\left(\begin{array}{c}
\left(6 e^{-t}-5 e^{-2 t}\right) \sin x+\left(6 \frac{e^{(1-t) m^{2}}}{m}-5 \frac{e^{2(1-t) m^{2}}}{m}\right) \sin (m x) \\
\left(-4 e^{-t}+5 e^{-2 t}\right) \sin x+\left(-4 \frac{e^{(1-t) m^{2}}}{m}+5 \frac{e^{2(1-t) m^{2}}}{m}\right) \sin (m x)
\end{array}\right)
$$


The data error at $t=1$,

$$
\left\|f_{m}(x)-f(x)\right\|^{2}=2 \int_{0}^{\pi} \frac{1}{m^{2}} \sin ^{2}(m x) d x=\frac{2}{m^{2}} \frac{\pi}{2},
$$

Notice that

$$
\lim _{m \rightarrow+\infty}\left\|f_{m}(x)-f(x)\right\|=\lim _{m \rightarrow+\infty} \frac{2}{m} \sqrt{\frac{\pi}{2}}=0,
$$

but

$$
\lim _{m \rightarrow+\infty}\left\|U_{m}(0, x)-U(0, x)\right\|=\lim _{m \rightarrow+\infty} \frac{e^{m^{2}}}{m} \sqrt{\frac{\pi}{2}}=\infty .
$$

Thus, arbitrarily small data errors can lead to arbitrarily large errors in the result. By applying the quasi-boundary value method, the regularized solution is given by

$$
\begin{gathered}
U_{\alpha}(t, x)=\sum_{j=1}^{+\infty}\left(\frac{e^{-t \lambda_{j}^{2}}}{\left(e^{-\lambda_{j}^{2}}+\alpha\right)} \mathcal{Q}_{1}+\frac{e^{-2 t \lambda_{j}^{2}}}{\left(e^{-2 \lambda_{j}^{2}}+\alpha\right)} \mathcal{Q}_{2}\right) P_{j} f, \\
U_{\alpha}(t, x)=\left(\begin{array}{c}
u_{1}^{\alpha}(t, x) \\
u_{2}^{\alpha}(t, x)
\end{array}\right) \\
=\left(\begin{array}{c}
\left(6 \frac{e^{-(1+t)}}{\left(\alpha+e^{-1}\right)}-5 \frac{e^{-2(1+t)}}{\left(\alpha+e^{-2}\right)}\right) \sin x+\frac{1}{m}\left(6 \frac{e^{-t m^{2}}}{\left(\alpha+e^{-m^{2}}\right)}-5 \frac{e^{-2 t m^{2}}}{\left(\alpha+e^{-2 m^{2}}\right)}\right) \sin m x \\
\left(-4 \frac{e^{-(1+t)}}{\left(\alpha+e^{-1}\right)}+5 \frac{e^{-2(1+t)}}{\left(\alpha+e^{-2}\right)}\right) \sin x+\frac{1}{m}\left(-4 \frac{e^{-t m^{2}}}{\left(\alpha+e^{-m^{2}}\right)}+5 \frac{e^{-2 t m^{2}}}{\left(\alpha+e^{-2 m^{2}}\right)}\right) \sin m x
\end{array}\right)
\end{gathered}
$$

It follows that

$$
\begin{aligned}
& U_{\alpha}\left(\frac{1}{2}, x\right)= \\
& \left(\begin{array}{c}
\left(6 \frac{e^{-\frac{3}{2}}}{\left(\alpha+e^{-1}\right)}-5 \frac{e^{-3}}{\left(\alpha+e^{-2}\right)}\right) \sin x+\frac{1}{m}\left(6 \frac{e^{-\frac{m^{2}}{2}}}{\left(\alpha+e^{-m^{2}}\right)}-5 \frac{e^{-m^{2}}}{\left(\alpha+e^{-2 m^{2}}\right)}\right) \sin m x \\
\left(-4 \frac{e^{-\frac{3}{2}}}{\left(\alpha+e^{-1}\right)}+5 \frac{e^{-3}}{\left(\alpha+e^{-2}\right)}\right) \sin x+\frac{1}{m}\left(-4 \frac{e^{-\frac{m^{2}}{2}}}{\left(\alpha+e^{-m^{2}}\right)}+5 \frac{e^{-m^{2}}}{\left(\alpha+e^{-2 m^{2}}\right)}\right) \sin m x
\end{array}\right) .
\end{aligned}
$$

If we choose $n=300$, the error of the quasi-boundary value method is given in the table 1 .

Table 1 shows that the approximate solutions converge to the exact solution as epsilon tends to zero. 


\begin{tabular}{|c|c|c|}
\hline$\alpha$ & $U_{\alpha}=\left(u_{1}^{\alpha}, u_{2}^{\alpha}\right)^{\top}$ & $b_{\alpha}=\left\|U_{\alpha}\left(\frac{1}{2},.\right)-U\left(\frac{1}{2},.\right)\right\|$ \\
\hline $10^{-2} \sqrt{\frac{\pi}{2}}$ & $\begin{array}{l}(1,83579453285757870327 \sin x+ \\
8,93890683320693679259 \times 10^{-19544} \sin (300 x), \\
-0,66269894986764767505 \sin x \\
\left.-5,95927122213795786173 \times 10^{-19544} \sin (300 x)\right)^{\top}\end{array}$ & 0,10537186355054932809 \\
\hline $10^{-4} \sqrt{\frac{\pi}{2}}$ & $\begin{array}{l}1,80024920972532375106 \sin (x)+ \\
8,93890683320693679259 \times 10^{-19542} \sin (300 x), \\
-0,58760102268700560842 \sin x \\
\left.-5,95927122213795786173 \times 10^{-19542} \sin (300 x)\right)^{\top}\end{array}$ & 0, 0012410493990990877 \\
\hline $10^{-8} \sqrt{\frac{\pi}{2}}$ & $\begin{array}{l}1,7997867987796889995 \sin x+ \\
8,93890683320693679259 \times 10^{-19538} \sin (300) x, \\
-0,58760102268700560842 \sin x \\
\left.-5,95927122213795786173 \times 10^{-19542} \sin (300) x\right)^{\top}\end{array}$ & $\begin{array}{l}1,24315933188250963919 \\
\times 10^{-7}\end{array}$ \\
\hline $10^{-16} \sqrt{\frac{\pi}{2}}$ & $\begin{array}{l}1,79978675241858939726 \sin x+ \\
8,93890683320693679259 \times 10^{-19530} \sin (300) x, \\
-0,58672543299332296332 \sin x \\
\left.-5,95927122213795786173 \times 10^{-19530} \sin (300) x\right)^{\top}\end{array}$ & $\begin{array}{l}1,24315954313594822062 \\
\times 10^{-15}\end{array}$ \\
\hline
\end{tabular}

Table 1

\section{Conclusion}

In this paper, we considered a quasi boundary value method to solve an ill posed diffusion system. In the theoretical results, it was shown that under certain conditions stability estimate was obtained and convergence results were established. Meanwhile, the numerical results verified the efficiency of this method. 


\section{Acknowledgments}

The authors would like to thank the referees for their comments and suggestions on the manuscript.

\section{References}

[1] L.S. Abdulkerimov, Regularization of an ill-posed Cauchy problem for evolution equations in a Banach space, Azerbaidzan. Gos. Univ. Ucen. Zap. Fiz. Mat., 1 (1977), 32-36 (MR0492645) (in Russian).

[2] R. Aris, The mathematical theory of diffusion and reaction in permeable catalysts, Clarendon Press, Oxford (1976). DOI: 10.1137/1018132.

[3] D.G. Aronson, The role of diffusion in mathematical population biology, Lecture Notes in Biomathematics, vol. 57 (1985), 2-6. DOI:10.1007/978 $3-642-93287-8 \_1$.

[4] G. W. Clark, S. F. Oppenheimer, Quasi-reversibility methods for non- well posed problems, Electronic Journal of Differential Equations, vol. 1994, No. 8 (1994) 1-9.

[5] A.D. Cliff, P. Haggett, J.K. Ord, G.R. Versey, Spatial Diffusion. An Historical Geography of Epidemics in an Island Community. Cambridge University Press, Cambridge (1981).

[6] P.C. Fife, Mathematical Aspects of Reacting and Diffusing Systems. Lecture Notes in Biomathematics, vol. 28, Springer-Verlag, Berlin, Heidelberg (1979). DOI: $10.1007 / 978-3-642-93111-6$.

[7] K.P. Hadeler, Diffusion Equations in Biology. Mathematics of Biology. vol. 80 (2011), 149-177. DOI: 10.1007/978 - 3-642 - $11069-6 \_3$

[8] V.K. Ivanov, I.V. Mel'nikova, A.I. Flinkov, Differential-operator equations and ill-posed problems, Nauka, Moscow (1994) (in Russian).

[9] H. Leiva, A lemma on C0-semigroups and applications PDEs systems, Quaestiones Mathematicae, vol. 26, No. 3 ( 2003), 247-265.

[10] L. F. de Oliveira, On reaction-diffusion systems. Electronic Journal of Differential Equations, vol. 1998, No. 24 (1998), 1-10. 
[11] I.V. Melnikova, A. I. Filinkov, Abstract Cauchy Problems : Three Approaches, Monographs and Surveys in Pure and Applied Mathematics, 120, Chapman and Hall, Boca Raton, FL (2001).

[12] I.V. Melnikova, Regularization of ill-posed differential problems, Siberian Math. J. 33 (1992), 289-298, DOI: 10.1007/BF00971100.

[13] A. Pazy, Semigroups of Linear Operators and Applications to Partial Differential Equations. Springer-verlag New York,(1983). DOI: 10.1007/978$1-4612-5561-1$.

[14] R. E. Showalter, Cauchy problem for hyper-parabolic partial differential equations, Trends in the theory and practice of non-linear analysis, NorthHolland Math. Stud., vol. 110 (1985), 421-425. DOI: 10.1016/S0304 0208(08)72739- 7 .

[15] P.N. Vabishchevich, Non-local parabolic problems and the inverse heatconduction problem, Differentsial'nye Uravnenija, 17 (1981), 1193-1199 (Russian); English transl. Differential Equations, 17 (1981), 761-765.

[16] I. Vrabie , CO -semigroups and Applications, North-Holland Publishing, Amsterdam (2003). 